\title{
„Bürgernahe Effizienz“ - 44. Richterwoche des Bundessozialgerichts
}

\section{BEATRIX TISCHLER}

Beatrix Tischler ist Richterin am Sozialgericht und zurzeit Wissenschaftliche Mitarbeiterin am Bundessozialgericht in Kassel

\begin{abstract}
Während der diesjährigen Richterwoche des Bundessozialgerichts (BSG) vom 6. bis 8. November 2012 wurden aktuelle Fragen zum Generalthema „Bürgernahe Effizienz" beleuchtet und diskutiert. Dabei wurde der Bogen von der Zertifizierung von Maßnahmen und Produkten, über die Akkreditierung von Stellen, den einstweiligen Rechtschutz im sozialgerichtlichen Verfahren bis hin zur Entschädigung bei überlangen Gerichtsverfahren gespannt. Den Schlusspunkt setzte die Aktuelle Stunde zum Thema „Armutsbekämpfung und Rente“.
\end{abstract}

Im Rahmen seiner Eröffnungsrede konnte der Präsident des Bundessozialgerichts, Peter Masuch, rund 400 Teilnehmer an der 44. Richterwoche des Bundessozialgerichts begrüßen. Nach Grußworten des Parlamentarischen Staatssekretärs bei der Bundesministerin für Arbeit und Soziales, Dr. Ralf Brauksiepe, des Staatssekretärs im Hessischen Ministerium der Justiz, für Integration und Europa, Dr. Rudolf Kriszeleit und dem Oberbürgermeister der Stadt Kassel, Bertram Hilgen, folgte mit der Festrede von Professor Dr. Heribert Prantl ein erster Höhepunkt der jährlich stattfindenden Richterwoche. Prantl hatte sich für seinen Vortrag selbst das Thema „Heimat Europa. Warum die EU sozial werden muss.“ gewählt. Dabei wies er zunächst darauf hin, dass für viele Bürger die EU derzeit das Gegenteil von „Bürgernaher Effizienz“ sei. Im Rahmen seiner Analyse des europäischen Einigungsprozesses bewertete Prantl Europa zwar schlussendlich als das Beste, was den Nationalstaaten je passiert sei, forderte aber gleichzeitig europäische Regeln für eine sozialverträgliche Wirtschaft, um den inneren Frieden in Europa zu sichern. Insoweit sei es auch am Europäischen Gerichtshof, sich von seinem Verständnis als Gerichtshof der Europäischen Wirtschaftsgemeinschaft, der allein auf Grund wirtschaftlicher Belange entscheide und dem das Verständnis für soziale Gerechtigkeit fehle, zu verabschieden. Solange Europa allein durch die Brille der Wettbewerbsfreiheit betrachtet werde, bleibe der Mensch auf der Strecke. Die Europäische Einigung könne vielmehr nur dann gelingen, wenn man die Erfolgsgeschichte der Sozialstaatlichkeit in den Nationalstaaten auf europäischer Ebene fortsetze. Prantl forderte ein Europa, dem die Menschen vertrauen können und durch das sie sich vor Lebenskrisen geschützt sehen. Prantl schloss mit einer Abwandlung der Präambel der Schweizer Bundesverfassung: Die Stärke Europas messe sich am Wohl der Schwachen und am Vertrauen der Bürger in Europa.

\section{Konformitätsbewertung und Akkreditierung}

Der Nachmittag des 6. November war der Akkreditierung und Zertifizierung gewidmet und wurde von einem der Geschäftsführer der Deutschen Ak- 
kreditierungsstelle GmbH, Dr. Frank Salchow eröffnet. Im Rahmen seines Vortrags „Konformitätsbewertung und Akkreditierung - Leistungen, Grenzen und Anwendungen“ erläuterte Salchow zunächst, dass das ursprünglich aus der Wirtschaft stammende System der Konformitätsbewertung seine heutige Bedeutung durch das New Approach (Neue Konzept) der EU aus den 80-er Jahren erlangt habe. Ziel sei es gewesen, den Binnenmarkt durch den Abbau von Handelshemmnissen zu fördern, indem Eingriffe des Staates auf das erforderliche Maß beschränkt werden. Hierzu sei ein Konformitätsbewertungssystem durch akkreditierte Stellen genutzt worden. Das New Approach sei 2008 im New Legislation Framework (Neuen Rechtsrahmen) fortgeschrieben worden, dessen wesentliche Bestandteile die Verordnungen (EG) Nr. 764 und $765 / 2008$ sowie der Beschluss (EG) Nr. $768 / 2008$ seien. Diese definierten die Konformitätsbewertung als Verfahren zur Bewertung, ob spezifische Anforderungen an ein Produkt, ein Verfahren, eine Dienstleistung, ein System oder eine Person erfüllt seien. Akkreditierung sei die Bestätigung durch eine nationale Akkreditierungsstelle, dass eine Konformitätsbewertungsstelle die in harmonisierten Normen festgelegten Anforderungen erfülle, um eine spezielle Konformitätsbewertungstätigkeit durchzuführen. In „Umsetzung“ des Neuen Rechtsrahmens sei in Deutschland 2009 die Deutsche Akkreditierungsstelle $\mathrm{GmbH}$ als nationale Akkreditierungsstelle errichtet worden, die am 1.10.2010 ihre Arbeit aufgenommen habe. Alle anderen bis dorthin in Deutschland tätigen Akkreditierungsstellen hätten zu diesem Termin ihre Tätigkeit einstellen müssen, da das Europarecht pro Mitgliedsstaat lediglich eine Akkreditierungsstelle zulasse. Die Akkreditierungsstelle begutachte Laboratorien, Inspektionsstellen und Zertifizierungsstellen für Produkte, Dienstleistungen, Personal und Systeme (z. B. Qualität, Umwelt). So seien in Deutschland derzeit rd. 4.400 Stellen akkreditiert. Aus dem Bereich des Sozialrechts seien hier die fachkundigen Stellen nach $\mathbb{S} 177$ Drittes Buch Sozialgesetzbuch (SGB III) zu nennen - die wiederum Träger und Maßnahmen zertifizieren. Als Leistung des seit 1.10.2010 geltenden Systems wertete Salchow insbesondere die regelmäßige
Überwachung der akkreditierten Stellen, die Unabhängigkeit der Akkreditierungsstelle, die Beteiligung der interessierten Kreise und die Veröffentlichung der Konformitätsbewertungsstellen mit ihren Kompetenzspektren. Als Grenzen des Systems monierte Salchow, dass der dokumentierte Stand der Technik oft veraltet und Überwachungsmaßnahmen nur angekündigt möglich seien. Schließlich stünden (kurzfristige) Sanktionsmöglichkeiten praktisch nicht zur Verfügung.

Anschließend referierte Prof. Dr. Andreas Spickhoff von der GeorgAugust-Universität in Göttingen zum Thema „Zur Zertifizierung im Medizinrecht - juristische Anmerkungen“. Spickhoff stellte einleitend allgemeine Überlegungen zur Sinnhaftigkeit von Akkreditierung und Zertifizierung als Verfahren der Qualitätssicherung an. Diese sei keineswegs pauschal zu bestreiten. Rechtliche und rechtspolitische Bedeutung erhielten Akkreditierung und Zertifizierung allerdings über ihren Einfluss auf Berufsfreiheit, Eigentumsgarantie und allgemeine Handlungsfreiheit. Wer - insbesondere das Verhältnis von Aufwand und Nutzen - kritisiere, errege den Verdacht, Qualitätssicherung in Frage zu stellen. Wer sich verweigere, habe mit zum Teil erheblichen Folgen für sein Handeln am Markt zu rechnen. Die den Zertifizierungsstellen nach erfolgter Zertifizierung noch zur Verfügung stehenden Handlungsinstrumente bewertete Spickhoff als defizitär. Insoweit sei die geplante Ausweitung der Kontrollmöglichkeiten durch unangekündigte Kontrollen zu begrüßen. Über die jüngsten Vorfälle im Zusammenhang mit mangelhaften Brustimplantaten schlug Spickhoff den Bogen zum Schwerpunkt seine Vortrags. Die Zertifizierung sei im Medizinproduktegesetz (MPG) nicht legal definiert. Aus dem Lateinischen könne man sich die Bedeutung „Sicherheit schaffen“ (certo facere) ableiten. Damit seien auch im Medizinprodukterecht Konformitätsbewertungsstellen, sog. Benannte Stellen, betraut. Deren Verhältnis zu den $\mathrm{Me}$ dizinprodukteherstellern werde überwiegend als privatrechtlich qualifiziert. Diese Bewertung werde zunächst auf die im Verhältnis zum Akkreditierungsverfahren unterschiedliche Terminologie gestützt. So werde im Rahmen des Akkreditierungsverfahrens auf öffentlich- rechtliche Begriffe wie Rücknahme oder Widerruf zurückgegriffen. Im Zertifizierungsverfahren hingegen beschreibe \18 MPG die Handlungsmöglichkeiten der Benannten Stelle mit „Einschränkung, Aussetzung und Zurückziehung von Bescheinigungen“. Auch der europarechtliche Hintergrund der Regelungen spreche für eine Zuordnung zum Privatrecht, da viele europäische Nachbarstaaten bereits eine Differenzierung zwischen Privat- und öffentlichem Recht nicht vornähmen. Wissenschaftlich ungeklärt seien bislang die Auswirkungen einer entsprechenden Zuordnung der „Einschränkung, Aussetzung und Zurückziehung von Bescheinigungen“ im Streitverfahren. In der Folge wandte sich Spickhoff der Frage der Reichweite von Zertifizierungen im Medizinprodukterecht anhand deren Verhältnis zu sozialrechtlichen Leistungspflichten zu. Im Unterschied zum Arzneimittelrecht kenne das Medizinprodukterecht keine Zulassungspflicht. Statt dessen setzten die Inbetriebnahme und das In-Verkehr-Bringen von Medizinprodukten eine CE-Zertifizierung und -Kennzeichnung voraus. Dabei läge der Zertifizierung ein Klassifizierungsverfahren zugrunde, dessen Abstufung sich am Gefährdungspotenzial des Medizinprodukts orientiere. Die Hersteller der Klasse 1-Medizinprodukte (geringstes Gefährdungspotenzial) seien berechtigt, ihre Produkte selbst zu zertifizieren, was im Hinblick auf die mangelnde Kontrolldichte bedenklich stimme. Im Anschluss vollzog Spickhoff das Verhältnis des Medizinprodukterechts und des Rechts der gesetzlichen Krankenversicherung an Hand der Darstellung der Rechtsprechung des Bundessozialgerichts und des Europäischen Gerichtshofs nach. Danach werde die Sicherheit und Eignung bzw. Funktionstauglichkeit von Medizinprodukten durch das Medizinprodukterecht und die CEZertifizierung präjudiziert. Diese Vorgaben der Rechtsprechung habe der Gesetzgeber in $\mathbb{S} 139$ Abs. 5 Fünftes Buch Sozialgesetzbuch (SGB V) umgesetzt. Die Präjudiz medizinprodukterechtlicher Sicherheit und Eignung gelte im Krankenversicherungsrecht allerdings nicht uneingeschränkt. Hiervon dürfe in Ausnahmefällen abwichen werden, wenn begründeter Anlass für Zweifel an Sicherheit und Eignung des Medizinprodukts bestehe. Solche könnten eher bei 
vom Hersteller als bei von der Benannten Stelle zertifizierten Medizinprodukten entstehen. Schließlich bedeute Präjudiz nicht gleichzeitig krankenversicherungsrechtliche Erstattungsfähigkeit der Kosten des Medizinprodukts. $\mathrm{Zu}$ dieser müsse die Aufnahme in das Hilfsmittelverzeichnis treten, für die wiederum weitere Anforderungen erfüllt sein müssen. Dabei könnten Aspekte ohne Bezug zur Verkehrsfähigkeit, wie medizinischer Nutzen, Produktinformation und Kosten berücksichtigt werden. Es sei also zu differenzieren zwischen Sicherheit und Eignung einerseits sowie den weiteren, nach dem Recht der Gesetzlichen Krankenversicherung vorgegebenen Voraussetzungen für die Aufnahme eines Medizinprodukts in das Hilfsmittelverzeichnis andererseits. Aussagen über die fehlende Eignung eines CE-gekennzeichneten Medizinprodukts seien zu vermeiden. In diesem Zusammenhang sei schließlich die $\mathrm{Zu}$ lässigkeit von Rahmenvereinbarungen mit über das Medizinprodukterecht hinausgehenden Vorgaben zum Qualitätsmanagement zu problematisieren. Abschließend vergewisserte Spickhoff noch einmal den Grundsatz der Tatbestandswirkung der CE-Zertifizierung auch für das Recht der Gesetzlichen Krankenversicherung. Ausnahmen seien nach $\mathbb{} 139$ Abs. 5 SGB V möglich und bedürften begründeter Zweifel. Die Tatbestandswirkung umfasse schließlich nicht die Bereiche, die nicht Gegenstand der Prüfung im Zertifizierungsverfahren seien.

\section{Einstweiliger Rechtsschutz im sozialgerichtlichen Verfahren}

Den zweiten Tag der Richterwoche eröffnete Dr. Jürgen Brand, Präsident des Landessozialgerichts Nordrhein-Westfalen a. D., mit seinem Vortrag zum Thema „Einstweiliger Rechtsschutz im sozialgerichtlichen Verfahren“. Der Referent ist aktuell anwaltlich tätig und betreut dabei speziell Verfahren im Bereich der sozialrechtlichen Betriebsprüfungen. Diese Tätigkeit prägte das Referat. Nach einem Exkurs zu den Anfängen des sozialgerichtlichen Eilrechtsschutzes im Anschluss an den Beschluss des Bundesverfassungsgerichts vom 19.10.1977 (Az. 2 BvR 42/76) und zur Verankerung normativer Vorgaben des Gesetzgebers in $\mathbb{S} \mathbb{S} 86 \mathrm{a}$ und b SGG zum 2.1.2002 stellte Brand kurz die systematischen und dogmatischen Zusammenhänge dar. Dabei ging er im Grundsatz davon aus, dass der Gesetzgeber ein „unperfektes Gesetz" geschaffen habe. Im Anschluss an diesen allgemeinen Überblick setzte Brand den Schwerpunkt seiner Darstellung beim behördlichen $(\mathbb{S} 86 \mathrm{a}$ Abs. 3 SGG) und gerichtlichen $(\mathbb{S} 86 \mathrm{~b}$ Abs. 1 SGG) Eilrechtsschutz in Anfechtungssachen. Dabei ging er davon aus, dass besondere Bedeutung den in \86a Abs. 2 Nr. 1 SGG geregelten Fallgruppen zukomme. Hier biete das Gesetz grundsätzlich keine Vorgaben zum Entscheidungsmaßstab. Auch soweit der Gesetzgeber in $\$ 86$ a Abs. 2 Nr. 1 SGG den Entscheidungsmaßstab für die behördliche Anordnung der aufschiebenden Wirkung regele, bleibe er eher vage, da „ernstliche Zweifel“ bzw. „unbillige Härten" Raum für mannigfaltige Interpretationen böten. Durch die Bezugnahme auf „unbillige Härten“ mache der Gesetzgeber aber deutlich, dass eine Abwägungsentscheidung zu treffen sei. Dies gelte für sämtliche behördliche und gerichtliche Eilrechtsschutzentscheidungen. Abzuwägen seien die Interessen der Allgemeinheit mit den Individualinteressen des Rechtsschutzsuchenden. Ein starres Prüfschema gebe es nicht. Alle denkbaren, sich aus $\mathbb{\$} 86 a$ Abs. 3 Satz 2 SGG ergebenden Kriterien seien einzustellen. Vorauszugehen habe eine zumindest summarische Prüfung der Tatsachenfragen. Bei Rechtsfragen könne eine abschließende Prüfung erfolgen. In der Folge wies der Referent noch darauf hin, welche Hürden Säumniszuschläge nach $\mathbb{} 24$ SGB IV im Verfahren des einstweiligen Rechtsschutzes darstellen können. Problematisch erscheine es weiter häufig, wenn das Gericht einstweiligen Rechtsschutz von der Beibringung einer Bankbürgschaft abhängig mache. Abschließend warb Brand dafür, bei der Streitwertfestsetzung für das Verfahren des einstweiligen Rechtsschutzes zu berücksichtigen, dass in der Praxis der sozialversicherungsrechtlichen Betriebsprüfung häufig das Verfahren des einstweiligen Rechtsschutzes an die Stelle des Hauptsacheverfahrens trete. Hier sei ein Abschlag auf den Hauptsachestreitwert nicht gerechtfertigt.

\section{Zur BSG-Rechtsprechung aus Sicht der Instanz}

Den Vormittag des zweiten Tages der Richterwoche komplettierte Dr. Christine Fuchsloch, Präsidentin des SchleswigHolsteinischen Landessozialgerichts, zum Thema „Stolpersteine und Hilfestellungen - Zur BSG-Rechtsprechung aus Sicht der Instanz“. Fuchsloch betonte bereits einleitend, dass es ihr bewusst nicht - in Anlehnung an ein Referat im Rahmen der Richterwoche 1998 - um einen „Blick hinauf im Zorn“ ginge. Sie habe ihre Einladung als Aufforderung zum Dialog zwischen den Instanzgerichten und dem BSG verstanden. Es ginge ihr darum, einerseits dem BSG die Sicht der Instanzgerichte näher zu bringen und andererseits für Verständnis zu werben. Diesem Ansatz folgend warf Fuchsloch dann zunächst die Frage auf „Was braucht die Instanz vom Revisionsgericht? “ und formulierte in der Folge die Erwartungshaltung der Sozial- und Landessozialgerichte an das Bundessozialgericht. Die Instanz wünsche die höchstrichterliche Beantwortung und Vereinheitlichung von Auslegungsfragen, keine Meinungsverschiedenheiten zwischen den einzelnen Fachsenaten, handhabbare Vorgaben zur Sachverhaltsermittlung und Verfahrenssteuerung sowie zurückhaltende und respektvolle Zurückverweisungsentscheidungen. Im weiteren erläuterte Fuchsloch diese Erwartungen näher. Dabei zeigte sie immer wieder Beispiele aus der Rechtsprechung des BSG auf, in denen diese Erwartungen erfüllt oder auch enttäuscht wurden. So definierte Fuchsloch unter dem Blickwinkel „Beantwortung und Vereinheitlichung von Auslegungsfragen“ als „Stolperstein“ eine höchstrichterliche Auslegung unbestimmter Rechtsbegriffe, die sich in der Heranziehung weiterer unbestimmter Rechtsbegriffe erschöpfe. Wenig hilfreich seien weiter Entscheidungen, deren Problemlösung nicht überzeuge. Als Beispiel hierfür nannte Fuchsloch insbesondere Entscheidungen, die zwar eine dogmatisch zutreffende Lösung präsentierten, jedoch nicht zur Rechtswirklichkeit passten und damit nicht praxistauglich seien. Gleichzeitig betonte die Referentin, dass die Rechtsprechung des BSG insoweit überwiegend Hilfestellung leiste, weil sie überzeugende Lösungen und durch stringente 
Ableitungen die Instanzrechtsprechung erleichtere. Hier sei besonders auf die gute Aufarbeitung und Zusammenfassung schwieriger rechtlicher Probleme in zahlreichen revisionsrechtlichen Entscheidungen hinzuweisen. Besonders hilfreich sei es, wenn rechtliche Hintergründe erklärt, Prüfungsschemata entwickelt und die bisherige Rechtsprechung - auch für den nicht täglich mit der Materie Befassten - aufgearbeitet würde. Problematisch und wenig hilfreich seien hingegen Meinungsverschiedenheiten zwischen den einzelnen Senaten des BSG. Hier entstünde bisweilen der Eindruck, dass Meinungsverschiedenheiten nicht offensichtlich genug herausgestellt würden, um eine Vorlage an den Großen Senat zu vermeiden. Fuchsloch bedauerte, dass der große Wunsch der Instanzgerichte nach „handhabbaren Vorgaben zur Sachverhaltsermittlung und zur Verfahrenssteuerung" gelegentlich vom Bemühen des BSG nach Einzelfallgerechtigkeit überlagert werde, wenn Anforderungen formuliert würden, die die Praxis überforderten. Ihre abschließenden Ausführungen zum Wunsch nach zurückhaltenden und respektvollen Zurückverweisungsentscheidungen leitete Fuchsloch zunächst mit dem Hinweis ein, dass es ihr nicht um die Zurückverweisung an sich gehe. Problematisch seien lediglich solche Entscheidungen, die den Eindruck vermitteln, dass das Revisionsgericht das Verfahren in der unteren Instanz nicht in guten Händen vermute. Positiv hob Fuchsloch die sinkende Tendenz der $\mathrm{Zu}$ rückverweisungsentscheidungen hervor. Resümierend stellte die Referentin fest, dass sich in den vergangenen Jahren viel geändert habe. Insoweit sei insbesondere die wachsende Kommunikationsfreude des BSG zu loben, die sich nicht zuletzt in den Jahrespressegesprächen, dem Internetauftritt mit seinen zahlreichen Informationen und schließlich der Richterwoche zeige, die es in dieser Form in keiner anderen Gerichtsbarkeit gebe. Fuchsloch begrüßte die Teilnahme der Bundesrichter an Fachtagungen, im Rahmen derer auch immer wieder die Gelegenheit genutzt werde, BSG-Rechtsprechung zu erläutern. Abschließend wünschte sich die Referentin weiteren Dialog zwischen den Instanzen, im Rahmen dessen sie sich schließlich einen Vortrag bei der nächsten Richterwoche zum Thema „Die instanzgerichtliche
Rechtsprechung aus Sicht des BSG“ vorstellen könne.

Der Nachmittag des 7. November gehörte den Arbeitsgemeinschaften, im Rahmen derer das BSG seine Rechtsprechung insbesondere seit der letzten Richterwoche darstellte und, soweit es die Zeit zuließ, Raum zur Diskussion aktueller Fragen bestand.

\section{Verfahrensdauer und Entschädigung}

Den dritten und letzten Tag der 44. Richterwoche eröffnete Lutz Wehrhahn, Vorsitzender Richter am Thüringer Landessozialgericht, mit seinem Vortrag zum Thema „Verfahrensdauer und Entschädigung“. Im Mittelpunkt des Vortrags stand das zum 3.12.2011 in Kraft getretene Gesetz über den Rechtsschutz bei überlangen Gerichtsverfahren und strafrechtlichen Ermittlungsverfahren (BGBl. I 2011 S. 2302 -2312), zum dem zwischenzeitlich erste Verfahren bei den erstinstanzlich zuständigen Landessozialgerichten anhängig sind. Einleitend wies der Referent darauf hin, dass die Dauer der sozialgerichtlichen Verfahren seit einigen Jahren immer mehr in den Fokus der Rechtsprechung des Europäischen Gerichtshofs für Menschenrechte (EGMR) und des Bundesverfassungsgerichts (BVerfG) getreten sei. Dabei sei die Verfahrensdauer dann als kritisch angesehen worden, wenn sie faktisch den $\mathrm{Zu}$ gang zu effektivem Rechtsschutz nach Art. 19 Abs. 4 GG nicht mehr gewährleistet habe. Mit dem Gesetz über den Rechtsschutz bei überlangen Gerichtsverfahren und strafrechtlichen Ermittlungsverfahren habe der Gesetzgeber auf eine sog. Pilotentscheidung des EGMR (Urteil vom 2.9.2010 - 46344/06) reagiert und sich dabei für eine Mischung aus präventiven und kompensatorischen Elementen entschieden. Hinsichtlich der einzelnen Tatbestandsmerkmale sei an die Rechtsprechung des EGMR und des BVerfG angeknüpft worden. In der Folge beschrieb Wehrhahn das für den Bereich der Sozialgerichtsbarkeit von $\$ 202$ SGG in Bezug genommene in den $\mathbb{S} \mathbb{S} 198$ bis 201 GVG geregelte Entschädigungsverfahren. Prüfungsgegenstand sei das „Gerichtsverfahren“ und zwar sämtlicher Instanzen, soweit sie in die Haftungsverantwortung des in Anspruch genommenen Rechtsträgers fallen. Ausgangspunkt sei dessen Einleitung und Endpunkt dessen rechtskräftiger Abschluss. Widerspruchsverfahren seien kein Gerichtsverfahren im genannten Sinn. Hier gehe der Gesetzgeber weiter davon aus, über die Untätigkeitsklage nach $\int 88$ SGG ausreichenden Rechtsschutz gegen eine überlange Dauer zur Verfügung zu stellen. Anspruchsteller im Entschädigungsverfahren könne jeder Verfahrensbeteiligte sein mit Ausnahme von Verfassungsorganen und öffentlichen Stellen. Das präventive Element sei die Verzögerungsrüge. Dabei handele es sich um eine Obliegenheit des Beteiligten, dem Richter ähnlich einer Mahnung ein Warnsignal zu senden, wenn die objektiven Verhältnisse erwarten lassen, dass das Verfahren nicht in angemessener Zeit abgeschlossen werde. Der Beteiligte müsse deutlich zum Ausdruck bringen, mit der Verfahrensdauer nicht einverstanden zu sein. Ob dies der Fall sei, müsse erforderlichenfalls durch Auslegung ermittelt werden. Das Erheben der Verzögerungsrüge sei schließlich materiell-rechtliche Voraussetzung für das Entstehen eines Entschädigungsanspruchs. Der Entschädigungsanspruch, der in den Gesetzesmaterialien als „staatshaftungsrechtlicher Anspruch sui generis“ bezeichnet werde, bilde das kompensatorische Element. Voraussetzung für einen Entschädigungsanspruch sei unter anderem die „unangemessene Dauer eines Gerichtsverfahrens“. Dabei habe der Gesetzgeber zur Bestimmung der Unangemessenheit auf die Rechtsprechung des EGMR und des BVerfG zurückgegriffen, wonach keine starren Fristen gelten sondern die Umstände des Einzelfalls zu bewerten seien. Ein Rückgriff auf Richtwerte und Statistiken verbiete sich deshalb. Vielmehr habe eine umfassende Abwägung an Hand der (tatsächlichen und/oder rechtlichen) Schwierigkeit und Bedeutung der Sache zu erfolgen, wobei letztere durch das Interesse bestimmt werde, Verzögerungen zu vermeiden. Soweit Umstände nicht der Sphäre des Staates zuzuordnen seien, könnten sie eine entschädigungsrelevante Verzögerung nicht begründen. Dabei sei aber auch zu berücksichtigen, dass es in jedem Stadium des Verfahrens Sache des Gerichts sei, die ihm zur Verfügung stehenden Mittel zur Verfahrensbeschleunigung z. B. durch das Setzen von Fristen einzusetzen. Entschädigt werden könne sowohl materieller als auch immaterieller Schaden, nicht 
hingegen entgangener Gewinn. Auch die $\mathbb{S} \int 249$ ff. BGB fänden keine Anwendung. Der Eintritt eines materiellen Schadens sei zu beweisen. Der Eintritt eines immateriellen Schadens hingegen werde vermutet. Insoweit setze das Gesetz pauschal $1.200 €$ jährlich an. Eine Abweichung hiervon sei, so Wehrhahn, nur in atypischen Fällen möglich. Neben der Verurteilung zur Entschädigung sehe das Gesetz die Feststellung der unangemessenen Verfahrensdauer durch das Gericht vor. Letztere komme isoliert in Betracht, wenn zwar eine überlange Verfahrensdauer nicht aber die übrigen Voraussetzungen für eine Entschädigung vorlägen. Die Feststellung unangemessener Verfahrensdauer sei weiter in besonders schwerwiegenden Fällen zusammen mit einer Verurteilung zur Entschädigung möglich. Verfahrensrechtlich wies Wehrhahn darauf hin, dass die Klage auf Entschädigung gegen den Träger der Justizhoheit zu richten und frühestens sechs Monate nach Erhebung der Verzögerungsrüge zulässig sei. Gleichzeitig müsse die Klage spätestens sechs Monate nach rechtskräftigem Abschluss des „überlangen“ Verfahrens erhoben sein. Das Entschädigungsverfahren sei schließlich auch dann gerichtskostenpflichtig, wenn das Ausgangsverfahren gerichtskostenfrei gewesen sei. In seinem Schlusswort zeigte sich der Referent überzeugt, dass der Gesetzgeber die Vorgaben des EGMR und des BVerfG auch unter Berücksichtigung der richterlichen Unabhängigkeit ausgewogen umgesetzt habe. Soweit der Gesetzgeber noch ausreichend Möglichkeiten zur Verfahrensbeschleunigung durch die Gerichte sehe, übersehe er allerdings, dass dies ohne vorherige Lösung der strukturellen Probleme in den Justizhaushalten nur auf Kosten der Qualität der gerichtlichen Entscheidungen möglich sei.

\section{Armutsbekämpfung und Rente}

Ihren Ausklang fand die 44. Richterwoche mit einer vom Präsidenten des Bundessozialgerichts moderierten Aktuellen Stunde zum Thema „Armutsbekämpfung und Rente“. Einleitend wies Masuch auf die Bedeutung dieses Anliegens hin und appellierte, den derzeit geführten „Kampf um das Recht“ in die „Armutsbekämpfung durch Recht“ zu führen.
Dr. Clarita Schwengers, Leiterin des Referats Koordination Sozialpolitik beim Deutschen Caritasverband e.V., führte in das Thema ein, indem sie sich kritisch mit den geplanten Maßnahmen zur Alterssicherung auseinandersetzte und die von Altersarmut betroffenen Personengruppen in den Vordergrund stellte. Nach einem kurzen Blick auf die derzeit bestehende Situation, bei der neben den bekannten Zahlen die verdeckte Armut nicht in Vergessenheit geraten dürfe, richtete sie den Fokus auf die zukünftige Entwicklung. Diese sei allgemein geprägt durch den demographischen Wandel, die Absenkung des Rentenniveaus sowie den Abschied von der Lebensstandardsicherung allein durch die gesetzliche Rentenversicherung. Diese Rahmenbedingungen hätten zur Folge, dass eine immer größer werdende Zahl von Versicherten von Altersarmut bedroht werde. Dies gelte vor allem für Menschen, die im Niedriglohnsektor und in geringfügigen Beschäftigungsverhältnissen tätig seien, die über eine brüchige Erwerbsbiographie verfügten sowie für Frauen, die häufig mit der Erziehung von Kindern und der Pflege von Angehörigen betraut seien. Um diese Personengruppen vor Altersarmut zu schützen, seien die derzeit vorgesehenen Regelungen nicht ausreichend. Schwengers forderte daher eine Stärkung der präventiven Maßnahmen auf den Gebieten Bildung, Arbeitsförderung und Entlohnung, eine Erhöhung des Anreizes für private Altersvorsorge, eine Absenkung der Zugangsvoraussetzungen für die Lebensleistungsrente, insbesondere für Menschen mit brüchiger Erwerbsbiographie, sowie eine bessere Berücksichtigung der Zeiten für Erziehung und Pflege.

Im Anschluss hieran betrachtete Richter am Bundessozialgericht Stefan Gasser die geplanten Alterssicherungsmaßnahmen aus richterlicher Sicht. Maßstab für die naturgemäß erst nach dem In-Kraft-Treten mögliche Prüfung, ob die entsprechenden Gesetze mit übergeordnetem Recht übereinstimmten, seien der allgemeine Gleichheitssatz des Art. 3 Abs. 1 GG sowie die speziellen Gleichheitssätze der Art. 3 Abs. 2 und 3 Satz 2 GG. Er erläuterte insbesondere die Anforderungen des allgemeinen Gleichheitssatzes auf der Basis der aktuellen Rechtsprechung des BVerfG. Dieser verbiete einen gleichheitswid- rigen Begünstigungsausschluss, wobei grundsätzlich dem Gesetzgeber ein weiter Gestaltungsspielraum zukomme, der allerdings dann enger werde, wenn nach personen- und verhaltensbezogenen Merkmalen differenziert werde oder die Gefahr der Diskriminierung einer Minderheit zu befürchten sei. Hinsichtlich der Zugangsvoraussetzungen für die Lebensleistungsrente wies Gasser darauf hin, dass bei Massenerscheinungen grundsätzlich typisierende, pauschalierende und generalisierende Regelungen zulässig seien; es dürfe allerdings kein atypischer Fall zum Leitbild erhoben werden. Zudem müsse bei der Umsetzung eines Konzepts das Gebot der Folgerichtigkeit beachtet werden, insbesondere dürften Schutzbedürftige nicht sachwidrig aus dem Empfängerkreis ausgeschlossen werden. Im Rahmen der Rechtfertigung einer gegebenenfalls bestehenden Ungleichbehandlung dürfe auf finanzpolitische Belange keine Rücksicht genommen werden. Abschließend hinterfragte Gasser kritisch, ob die Anerkennung der Lebensleistung einen geeigneten Rechtfertigungsgrund darstellen könne.

Nach dieser Darstellung aus der Sicht der Judikative erläuterte Ministerialdirektor Christian Luft, Leiter der Abteilung Sozialversicherung, Alterssicherung, Sozialhilfe im Bundesministerium für Arbeit und Soziales (BMAS), die aktuellen Gesetzgebungsvorhaben. Er ging hierbei zunächst kurz auf das geplante Beitragssatzgesetz 2013 ein, wonach der Beitrag zur gesetzlichen Rentenversicherung ab 1.1.2013 auf $18,9 \%$ sinken solle. Die gesetzliche Rentenversicherung weise eine solide Finanzierung auf. Zudem handele es sich bei der demographischen Entwicklung um ein langfristiges Problem, das nicht durch eine größere Schwankungsreserve zu lösen sei. Sodann stellte er das beabsichtige Rentenpaket dar, insbesondere die Lebensleistungsrente, wozu es seit den Beschlüssen des Koalitionsausschusses vom 4.11.2012 einige Klarstellungen gebe. Es handele sich hierbei um ein langfristig gedachtes und angelegtes Vorhaben, das zum Ziel habe, dass Menschen, die ihr Leben lang versicherungspflichtig beschäftigt gewesen seien, besser dastünden, als Menschen, die weniger gearbeitet hätten. Werde dieses Ziel nicht verfolgt, stehe die gesetzliche Rentenversicherung vor einem Legitima- 
tionsproblem. Luft wies zudem darauf hin, dass es sich bei den Berechtigten der Lebensleistungsrente um eine kleine Zielgruppe handele; eine gesamte Lösung des Problems der Altersarmut sei auf diesem Wege daher nicht zu erreichen. Hierfür müssten die Ursachen der Altersarmut angegangen werden wie z. B. die Bereiche Bildung oder Entlohnung, wofür jedoch das BMAS nicht zuständig sei.

Abschließend bewertete Michael Schröter, Referent Sozialpolitik gegen Ausgrenzung und Armut bei der Diakonie Deutschland, die zentralen Probleme der Alterssicherung sowie die angedachten Maßnahmen der Bundesregierung. Diese Maßnahmen seien ein erster Schritt in die richtige Richtung; es gebe jedoch weiterhin Handlungsbedarf. Die bereits erwähnten Beschlüsse des Koalitionsausschusses brächten nur vereinzelte Verbesserungen, zu einem großen Teil blieben sie unklar und unverbindlich. Geeigneter als eine Aufstockung der Rentenleistungen sei eine gesetzliche Mindestrente für langjährig Versicherte, die einfach konzipiert sei, wenig Bürokratie bedürfe sowie steuerfinanziert sei. Hierdurch solle erreicht werden, dass weniger Menschen Leistungen der Grundsicherung im Alter beziehen müssten. Er kritisierte die geplante Absenkung des Beitragssatzes sowie die vorgesehene Kürzung des Bundeszuschusses. Der in $\mathbb{} 158$ Abs. 1 Satz 1 Nr. 2 SGB VI angelegte „QuasiAutomatismus“ der Beitragsabsenkung müsse abgeschafft werden, da hierdurch die Kosten der Bekämpfung der Altersarmut in die Zukunft verschoben würden. Das Erfordernis der zusätzlichen privaten Altersvorsorge sei für viele Personengruppen, etwa für Beschäftigte im Niedriglohnbereich, nicht zu erfüllen, ohne ihr Existenzminimum anzugreifen. Hier müssten verstärkt Anreize geschaffen und Informationsmöglichkeiten verbessert werden. Schröter schloss seinen Vortrag mit der Forderung nach Präventionsmaßnahmen, um Altersarmut gar nicht erst entstehen zu lassen.

Im Anschluss an die Aktuelle Stunde schloss der Präsident des Bundessozialgerichts die Tagung verbunden mit der Ankündigung der 45. Richterwoche vom 5. bis 7. November 2013.

\section{Forschung und Entwicklung in der Sozialwirtschaft}

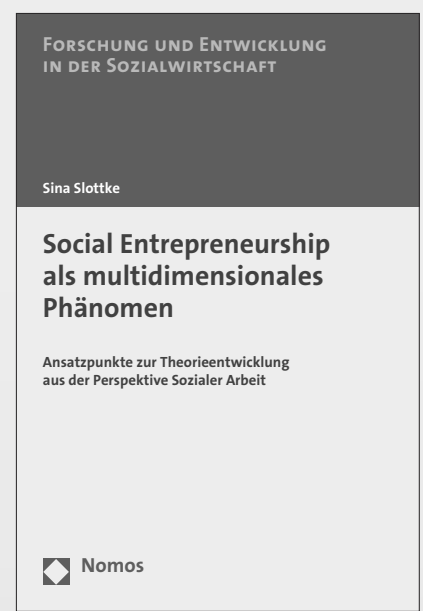

\section{Social Entrepreneurship als multidimensionales Phänomen}

Ansatzpunkte zur Theorieentwicklung aus der Perspektive Sozialer Arbeit

Von Sina Slottke

2013, Band 7, 104 S., brosch., 19,- $€$

ISBN 978-3-8487-0069-1

Soziale Arbeit als Human Rights Profession erweitert die wirtschaftsund managementfokussierte Diskussion um Social Entrepreneurship als zukunftsweisendem Lösungsansatz sozialer Problemlagen um bisher unberücksichtigte inhaltliche und strukturelle Impulse und leistet somit einen Beitrag für die überfällige ethische Fundierung des populären Phänomens.

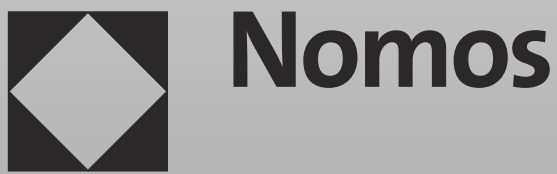

